Research Article

\title{
Molecular detection of HECV-4408 in Iraq
}

\begin{abstract}
This study was carried out to evaluate some epidemiological features of HECV infection by using real-time fluorogenic quantitative reverse transcription polymerase chain reaction assay based on SYBR Green I die in detection of CoVs. Both virus species (HECV-4408 and $\mathrm{BCoV}$ ) detected by the same neocleocapsid $(\mathrm{N})$ gene primer fewer than $98 \%$ similarity according to NCBI with product size $124 \mathrm{bp}$.

The technique has not been established for detection of HECV-4408 worldwide, and in detection of BCoVs in Iraq. One hundred fresh stool specimens were collected from hospitalized children between December 2012 to May 2013 in Maternal and Children's Hospital and dispensaries in districts and subdistricts of Al- Diawaniyia province, children showed acute diarrhea with some respiratory signs, all stool were examined by routine diagnosis methods against pathogenic bacteria (E. coli, Shigella, and Salmonella spp.) and parasitic invasion of Cryptosporidium spp.

The diagnostic examination of entero-pathagenic bacteria in fecal samples has been established just to take unidea about the occurrence of bacterial infection with coronavirus, while the positive cryptosporidium spp. samples submitted to molecular test as possible mix infection with coronavirus. HECV-4408 has been detected via reverse transcription realtime-qPCR in 18 out of 41 children's stool despite the infection with HCEV-4408 being found in the center of province and other districts was $50 \%-60 \%$, remains the highest percentage for the spreading of infection with the virus in rural areas up to $100 \%$. Infection percentage in females reached to $52.2 \%$ while in males lowered to $33.3 \%$. And $50 \%-57.1 \%$ of children infected with HECV-4408 they were at age $\geq 6$ years but the infection was low in neonates $15.3 \%$. The peak of HCEV-4408 activity often appeared during April $60 \%$ in comparison with January $50 \%$, March $46.7 \%$ and February $40 \%$.
\end{abstract}

Keywords: HECV-4408; Epidemiological features of HECV; Molecular detection of $\mathrm{CoVs}$
Volume 3 Issue I - 2016

Khetam Qaid Mayea AL-Hammdawy

AlQadissyia University, Iraq

Correspondence: Khetam Qaid Mayea AL-Hammdawy, AIQadissyia University, Vet. Med. College / Researches \& Zoonotic diseases unit, Iraq, Email dr_khokha_86@yahoo.com

Received: October 09, 2015 | Published: January 19, 2016

\section{Introduction}

CoVs are common human pathogens affecting children and adults worldwide with most individuals becoming infected in the first few years of life. ${ }^{1}$ And their role in enteric infections is less clear, even though CoVs-like particles have been seen by electron microscopy in stool samples from patients with diarrhea, they have been also found in healthy individuals. ${ }^{2}$

Generally, CoVs have been displayed marked winter seasonality between the months of December and April and were not detected in summer months, which are comparable to the pattern seen with influenza viruses, ${ }^{3}$ with a short incubation time, ${ }^{4}$ in temperate climates. ${ }^{5} \mathrm{HECV}-4408$ is discovered causative agent isolated firstly from a child with acute gastroenteritis, and it was antigenically and genetically more closely related to bovine coronavirus $(\mathrm{BCoV})$ than to human coronavirus $\mathrm{OC} 43 .{ }^{6}$

\section{Aims of study}

a. To evaluate some of risk factors of (HECV-4408) in diarrheic samples from children in Al-Qadissyia province, and analyze the association between infection regions, gender, age, and distribution in months.

b. To assessments the zoonotic importance of coronaviruses.

c. Specific rapid detection of Coronaviruses by using molecular method real time qRT-PCR in children.

\section{Materials and methods}

Primers A specific oligonucleotide primers set by using the complete sequence of Nucleocapsid gene of Human/Bovine Enteri
Coronaviruses GenBank No. (NC 003045.1) which have a similarity at present $98 \%$ according to NCBI plast analysis, using NCBI GeneBank and Primer 3 online and provided by (Bioneer Company, Korea) as following forward / ATTTGCAGAGGGACAAGGTG \& reveres / TAGCAATTGACGCTGGTTGC have 124bp product size Viral RNA was extracted from 41 children stools by using AccuZolTM Total RNA extraction kit (Bioneer, Korea) and done according to company instructions. cDNA synthesis a total RNA that extracted from stool samples were used in cDNA synthesis step by using AccuPower ${ }^{\circledR}$ RocktScript RT PreMix kit. Real-Time PCR master mix was prepared by Real-Time PCR detection kit (AccuPower $\rightarrow$ GreenStarTMqPCRPreMix).

\section{Results and discussion}

Based on the unique biological properties and the more distant relatedness of strains HECV-4408 / BCoVs, a universal published primer that had been developed to detect $\mathrm{BCoVs}$ and to determine the cross molecular -reactivity of HECV-4408, by targeting of the $\mathrm{N}$ gene (nucleocapsid gene) that was conserved among HECV-4408 / BCoV strains was used, As showed in the Figures (1-4). The important advantages of SYBR Green I over other real-time PCR detection formats are;

i. It is a low-cost fluorochrome

ii. It is a simpler approach especially for primer design and optimization procedures ${ }^{7}$

iii. The artifacts commonly observed in specific probes, particularly at amplification cycles beyond the 30th, are minimal and can be ruled out by melt curve analysis. ${ }^{8}$ 


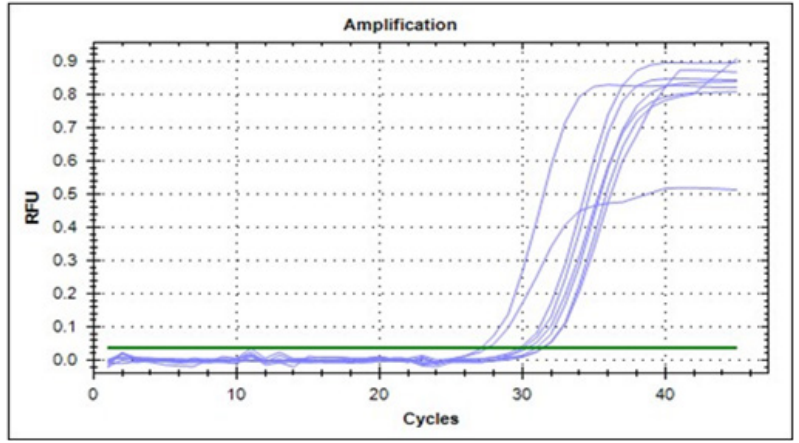

Figure I Reverse Transcription Real-Time PCR amplification plot shown the positive results of HECV-4408 in children samples.

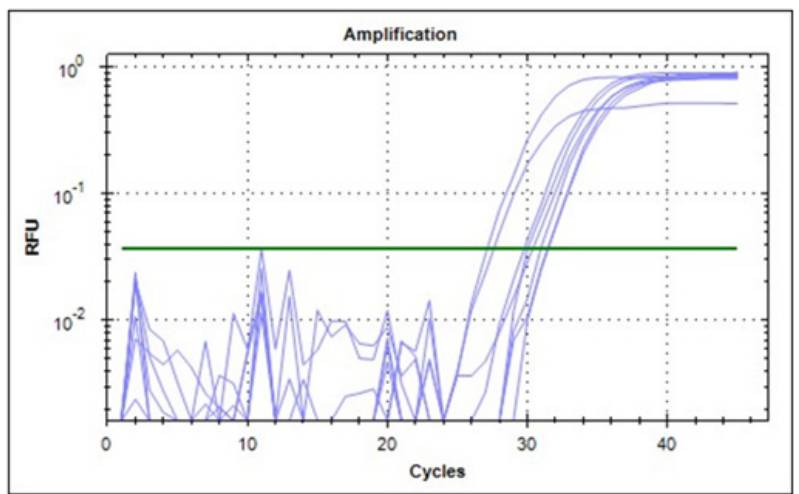

Figure 2 Reverse Transcription Real-Time PCR amplification log plot shown the positive results of HECV-4408 in children samples.

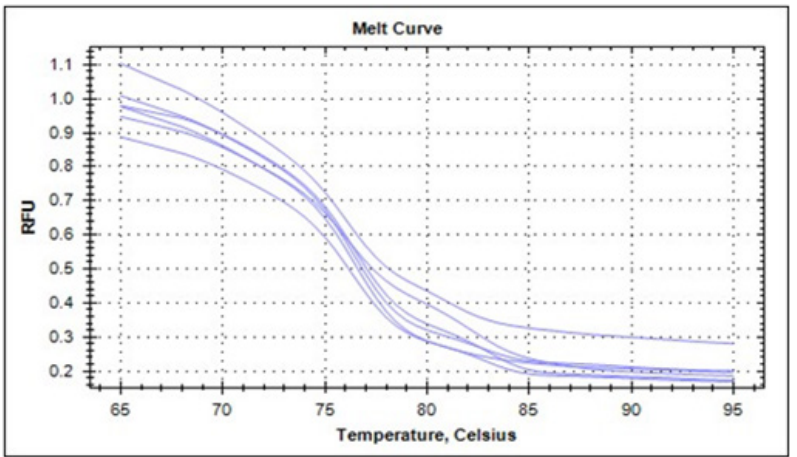

Figure 3 Reverse Transcription Real-Time PCR melt curve shown the positive results of HECV-4408 in children samples.

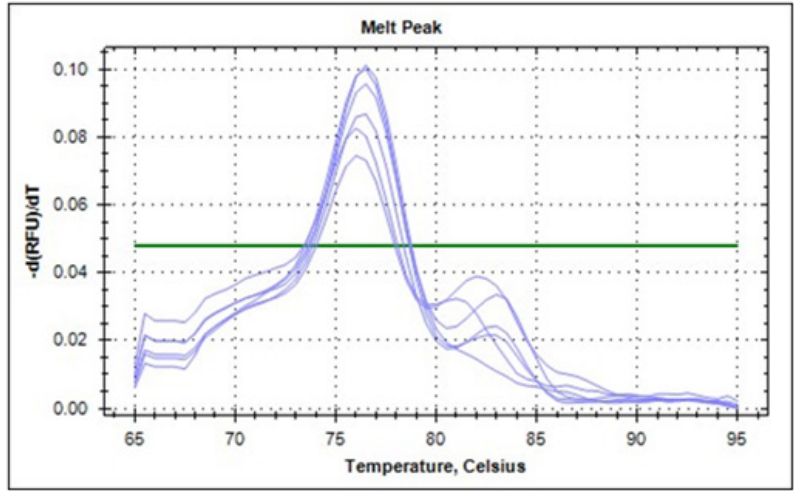

Figure 4 Reverse Transcription Real-Time PCR melt peak shown the positive results of Human enteric coronvirus- 4408 in children samples.
The results of molecular detection by Real time qRT-PCR of HECV-4408 in children according to study districts and regions in AL-Diwaniyia province, showed the total percentage of infection was 18 out of $41(43.9 \%)$. In districts city center, Hamza, Afak, and Shamyia were $50 \%, 60 \%, 60 \%, 50 \%$ respectively, the highest percent rate in Hamza and Afak $60 \%$ while the lowest percent rate incity center and Shamyia 50\%. In sub districts the results in Shafeyia, Albdeer, Dughara, and Sumer were 33.3\%, 25\%, 0, 100\%, respectively.

The highest percentage rate in Sumer 100\% whiles the lowest percentage rate in Albdeer 25\%. There was significant differences $(\mathrm{p}<0.05)$ between Shafeyia and Albdeer, Dughara, and Sumer, respectively. Between Albdeer and Sumer, Dughara respectively, between Sumer and Dughara. The positivity percentage of HECV4408 in districts was reached to $60 \%$. Whereas, in regions was higher to $100 \%$, this may indicate for a large number of children about $43.9 \%$ are faced with the danger of HECV-4408 infection associated diarrhea or respiratory syndrome. Increasing in the infection rate of HECV4408 within areas could be due to the fact that, peoples in rural areas keep touch with animal's population.

This finding is in agreements with the findings of that have been reported a group 2 human coronavirus designated HECV-4408 isolated from a child with acute diarrhea lived in a rural area with the opportunity for contact with cattle or manure. The results of HEV4408 infection according to children genders showed the highest positivity rate $52.2 \%$ was in females and the lowest positivity rate in males $33.3 \%$. Statistically there was a significant difference ( $\mathrm{p}<$ 0.05 ) between males and females. A study showed that, HECV-4408 infections in children, have a significant difference between males and females, the females showed highest positivity percentage $52.1 \%$, whereas $33.3 \%$ was the positivity percentage for males. In fact, in the case of viral infection in general, this difference in susceptibility between males and females was shown for different viruses. ${ }^{9}$ For many years the concept of sex-based (or gender based) differences in host response to infection has been studied and appears to be highly related to differences in immunological capacities between males and females. ${ }^{10}$

Furthermore, it appears now that even though the relative importance of different factors may vary with the type of infection, $\mathrm{X}$-linked genes, hormones, immunity and, at least in humans, societal context are among the factors that explain this sex-based difference.

Even though it remains difficult to clarify evidence on how the different factors make a difference between genders, studies aimed to addressing the question appear to more and more link specific hormones such as androgens in males and estrogens in females ${ }^{9,11}$ host innate immunity (expression of cytokines and of pattern recognition receptors such as toll-like receptors (TLR) $)^{12,13}$ as well as acquired immunity involving $\mathrm{T}$ and B lymphocytes (Klein, 2000) to explains, also the children in urban areas $42 \%$ are more likely to receive the recommended treatment than those living in rural areas 38\%. Similarly, children from the wealthiest households $40 \%$ are more likely to receive the recommended treatment than those from the poorest households $34 \%$ according to the reports of. ${ }^{14}$

The results of HECV-4408 infection according to the age groups showed that (1day_5months) and $(<5$ month_3 years, and $<3$ _6 years) were $15.3 \%, 57.1 \%$, and $50 \%$ respectively, the highest percent rate in childes aged $<5$ month 3 years $57.1 \%$ and the lowest percent rate was in 1day_5months. There were significant differences $(\mathrm{p}<$ 0.05 ) between children in ages 1 day 5 months and $<5$ month 3 years, $<3 \_6$ years respectively. No significant differences on $(-\bar{p}<0.05)$ between children in ages $<5$ month_3 years and $<3$ _6 years. 
The children included in our study were under six years of age, there was no significant differences between children $<5$ months-3years and $<3$ 6years, this results in agreements with ${ }^{15,16}$ they were proved that coronaviruses are common human pathogens affecting children and adults worldwide with most individuals becoming infected in the first few years of life. No study controlling for age and month of illness has demonstrated an epidemiologic association between infections with HECV-4408. But there is one reason for significant difference and the lowest positivity percentage at (1day-5 months) it was the presence of maternal antibodies directed to $\mathrm{HCoVs}$ in younger infants. ${ }^{17}$

The results showed that HECV-4408 infections are unequal distributed throughout cold and warm months, the highest percentage recorded in April 60\%, because human coronaviruses infections have also been strongly associated with seasonality distribution during warmer seasons in agreements with previously published studies. ${ }^{18,19}$ Followed by January $50 \%$. Those results agreed with ${ }^{2,5,20,21}$ they were proved that human coronavirus infections occur mainly in winter, with a short incubation time, that also explain the disappearing of viral infection evidence during December.

\section{Reverse transcription real time qRT-PCR results}

Amplification plots represent 8 positive samples; the results showed different positive reaction cycles of threshold $(\mathrm{Ct})$, they started reactions at $27,28,29$, and 30 respectively. The amplification started from the $\mathrm{Ct} 26$ and ended at 30 .

\section{Conclusion}

Syber Green I based real-time PCR is a sequence independent assay that requires conduction of a melt curve analysis on the PCR products. Specific amplification of the HECV-4408 target sequence was identified by the generation of a melt peak at 78.35 C 0.26 . Specificity of the reaction was further evaluated for cross reactivity against different viruses that show symptomatic or genetic relationship with HECV-4408.

\section{Acknowledgments}

None.

\section{Conflicts of interest}

None.

\section{References}

1. Dijkman R, Jebbink MF, Idrissi NB, et al. Human coronavirus NL63 and 229E seroconversion in children. $J$ Clin Microbiol. 2008;46(7):2368-2373.

2. Jevšnik M, Steyer A, Zrim T, et al. Detection of human coronaviruses in simultaneously collected stool samples and nasopharyngeal swabs from hospitalized children with acute gastroenteritis. J Virol. 2013;10:46

3. Gaunt ER, Hardie A, Claas EC, et al. Epidemiology and clinical presentations of the four human coronaviruses 229E, HKU1, NL63, and OC43 detected over 3 years using a novel multiplex Real-Time PCR method. J Clin Microbiol. 2010;48(8):2940-2947.

4. Gerna G, Campanini G, Rovida F, et al. Genetic variability of human coronavirus OC43-,229E-, and NL63-like strains and their association with lower respiratory tract infections of hospitalized infants and immunocompromised patients. J Med Virol. 2006;78(7):938-949.
5. Dare RK, Fry AM, Chittaganpitch M, et al. Human coronavirus infections in rural Thailand: a comprehensive study using real-time reverse-transcription polymerase chain reaction assays. $J$ Infect Dis. 2007;196(9):1321-1328

6. Zhang XM, Herbst W, Kousoulas KG, et al. Biological and genetic characterization of a hemagglutinating coronavirus isolated from a diarrhoeic child. J Med Virol. 1994;44(2):152-161.

7. Aldea C, Alvarez CP, Folgueira L, et al. Rapid detection of herpes simplex virus DNA in genital ulcers by real-time PCR using SYBR Green I dye as the detection signal. J Clin Microbiol. 2002;40(3):1060-1062.

8. Karlsen F, Steen HB, Nesland JM. SYBR Green I DNA staining increases the detection sensitivity of viruses by polymerase chain reaction. $J$ Virol Methods. 1995;55(1):153-156.

9. Klein SL. The effects of hormones on sex differences in infection: from genes to behavior. Neurosci Biobehav Rev. 2000;24(6):627-638.

10. Fish EN. The X-files in immunity: sex-based differences predispose immune responses. Nat Rev Immunol. 2008;8(9):737-744.

11. Wilson RP, White CR, Quintana F, et al. Moving towards acceleration for estimates of activity-specific metabolic rate in free-living animals: the case of the cormorant. J Anim Ecol. 2006;75(5):1081-1090.

12. Hannah MF, Bajic VB, Klein SL. Sex differences in the recognition of innate antiviral responses to Seoul virus in Norway rats. Brain Behave Immun. 2008;22(4):503-516.

13. Hill KE, Pigmans M, Fujinami RS, et al. Gender variations in early Theiler's virus induced demyelinating disease: differential susceptibility and effects of IL -4, IL-10 and combined IL -4 with IL-10. J Neuro Immunol. 1998;85(1):44-51.

14. UNICEF/WHO. Diarrhoea: why children are still dying and what can be done. 2009

15. Garbino J, Crespo S, Aubert JD, et al. A prospective hospital-based study of the clinical impact of non- severe acute respiratory syndrome (Non-SARS)-related human coronavirus infection. Clin Infect Dis. 2006;43(8):1009-1015.

16. Brittain-Long R, Westin J, et al. Prospective evaluation of a novel multiplex real-time PCR assay for detection of fifteen respiratory pathogens-duration of symptoms significantly affects detection rate. $J$ Clin Virol. 2010;47(3):263-267.

17. Dijkman R, Jebbink MF, Gaunt E, et al. The dominance of human coronavirus OC43 and NL63 infections in infants. $J$ Clin Virol. 2012;53(2):135-139.

18. Leung TF, Li CY, Lam WY, et al. Epidemiology and clinical presentations of human coronavirus NL63 infections in Hong Kong children. J Clin Microbiol. 2009;47(11):3486-3492.

19. Lau SK, Woo PC, Yip CC, et al. Coronavirus HKU1 and other coronavirus infections in Hong Kong. J Clin Microbiol. 2006;44(6):2063-2071.

20. Bastien N, Anderson K, Hart L, et al. Human coronavirus NL63 infection in Canada. J Infect Dis. 2005;191(4):503-506.

21. Arden KE, Nissen MD, Sloots TP, et al. New human coronavirus, $\mathrm{HCoV}-\mathrm{NL} 63$, associated with severe lower respiratory tract disease in Australia. J Med Virol. 2005;75(3):455-462. 